\title{
Imaging video plethysmography shows reduced signal amplitude in glaucoma patients in the area of the microvascular tissue of the optic nerve head
}

\author{
Ralf-Peter Tornow $^{1}$ (D) Radim Kolar $^{2}$ (D) $\cdot$ Jan Odstrcilik $^{2}$ (D) Ivana Labounkova $^{2}$ (D) Folkert Horn $^{1}$ (D)
}

Received: 2 April 2020 / Revised: 1 September 2020 / Accepted: 10 September 2020 / Published online: 22 September 2020

(C) The Author(s) 2020

\begin{abstract}
Purpose To measure parameters of the cardiac cycle-induced pulsatile light absorption signal (plethysmography signal) of the optic nerve head $(\mathrm{ONH})$ and to compare parameters between normal subjects and patients with different stages of glaucoma.

Patients and methods A recently developed video ophthalmoscope was used to acquire short video sequences (10 s) of the $\mathrm{ONH}$. After image registration and trend correction, the pulsatile changing light absorption at the ONH tissue (excluding large vessels) was calculated. The changing light absorption depends on the pulsatile changing blood volume. Various parameters, including peak amplitude, steepness, time-to-peak, full width at half maximum (FWHM), and pulse duration, were calculated for averaged individual pulses (heartbeats) of the plethysmography signal. This method was applied to 19 healthy control subjects and 91 subjects with ocular hypertension, as well as different stages of primary open-angle glaucoma (17 subjects with ocular hypertension, 24 with preperimetric glaucoma, and 50 with perimetric glaucoma).

Results Compared to the normal subjects, significant reductions $(\mathrm{p}<0.001)$ in peak amplitude and steepness were observed in the group of perimetric glaucoma patients, but no significant difference was found for time-to-peak, FWHM, and pulse duration. Peak amplitude and steepness showed high correlations with RNFL thickness $(\mathrm{p}<0.001)$.

Conclusions The presented low-cost video-ophthalmoscope permits measurement of the plethysmographic signal of the $\mathrm{ONH}$ tissue and calculation of different blood flow-related parameters. The reduced values of the amplitude and steepness parameters in perimetric glaucoma patients suggest decreased $\mathrm{ONH}$ perfusion and blood volume. This outcome is in agreement with results from other studies using OCT angiography and laser speckle flowgraphy, which confirm reduced capillary density in these patients.
\end{abstract}

Registration site: www.clinicaltrials.gov, Trial registration number: NCT00494923

Keywords Retinal plethysmography $\cdot$ Glaucoma $\cdot$ Perfusion $\cdot$ Blood volume $\cdot$ Blood flow

\section{Introduction}

Knowledge of the perfusion of the optic nerve head $(\mathrm{ONH})$ is important not only in the diagnosis of ocular diseases such as glaucoma and diabetic retinopathy but also in basic research to

Ralf-Peter Tornow

ralf.tornow@web.de

1 Department of Ophthalmology, Friedrich-Alexander-University of Erlangen-Nuremberg, 91054 Erlangen, Germany

2 Department of Biomedical Engineering, Faculty of Electrical Engineering and Communication, Brno University of Technology, 61600 Brno, Czech Republic better understand the aetiology of eye diseases. There is a huge variety of different methods to assess ocular blood flow (OBF) [1]. Techniques differ in the underlying basic principles, fields of view (e.g. macular region, $\mathrm{ONH}$, single vessels), measured location (large vessels or capillaries), and assessed parameters.

Optical methods to assess ocular blood flow are based on two basic principles: light absorption by blood components or light scattering by moving blood components.

In early measurements of retinal blood flow, non-imaging light absorption-based methods were used [2-6]. With light absorption-based methods, only the changing fraction of the blood flow can be measured. This changing fraction can occur due to provocation (increased intraocular pressure (IOP), flickering light, changing intensity after injection of dyes) 


\section{Key messages}

- The photoplethysmographic (PPG) principles is based upon light absorption due to the changing blood volume in the investigated tissue.

- We show that the PPG principles can be applied for assessment of optic nerve head perfusion with high spatial and temporal resolution using a previously published experimental ophthalmoscope and specific image and signal processing approaches.

- The amplitude and steepness parameters of the PPG signal are reduced in glaucoma patients and show a high statistically significant correlation with the RNFL thickness.

- The presented PPG analysis could give new insights into ocular blood flow.

and also due to the cardiac cycle-induced pulsatile changing blood volume in the investigated region of interest (ROI). With the development of highly light-sensitive imaging detectors, non-invasive imaging methods to measure cardiac cycleinduced changing blood volume could also be realized [7-11]. Examples of imaging dye injection-based methods are fluorescein and indocyanine green angiographies, two important techniques for clinical applications to visualize and assess ocular blood flow. However, due to the dye injection, these methods are invasive and their popularity is decreasing due to the development of new, advanced, dye-free imaging techniques. So far, non-invasive light absorption-based blood flow measurement methods have no clinical importance.

There are also methods that measure the movement of blood cells using coherent light and interference techniques such as the Doppler effect or speckle methods. Doppler techniques and speckle methods represent two ways of looking at the same physical phenomenon $[1,12,13]$. Scanning laser flowmetry (SLF), the combination of a laser Doppler flowmeter with a scanning laser system, allows visualization of the perfusion of the retina and ONH [14]. However, SLF has only slight clinical importance. Two non-invasive optical methods with a widespread clinical application are OCT angiography (OCT-A) and laser speckle flowgraphy (LSF). Both methods can be realized as imaging methods and are available as commercial instruments.

Optical coherence tomography angiography (OCT-A) is a functional extension of OCT that provides information/ visualization of perfused retinal (and choroidal) vasculature without the need for dye injection [15]. Blood flow-related parameters are calculated from several consecutive B-scans acquired at the same location. There are different algorithms to calculate parameters from raw data [16-20]. The most important parameters for clinical applications are capillary density and blood flow index [21]. Recent studies show that the OCT-A technique is able to clearly visualize perfusion changes in the microvascular network inside $\mathrm{ONH}$ and in the peripapillary area [16, 22-25]. However, the waveform of the pulsatile component of the blood flow cannot be assessed by OCT-A due to the relatively long acquisition time (several seconds).
Laser speckle flowgraphy (LSF) can also be applied to measure perfusion-related parameters. The method is based on changed speckle statistics for moving targets, which can be evaluated by computing the image contrast. The primary outcome is the variation in time of the mean blur rate (MBR), which is a dimensionless parameter depending on the erythrocyte velocity [26]. MBR shows a pulsatile behaviour that reflects the pulsatile blood flow. From the waveform of MBR, additional parameters can be calculated - average MBR $\left(\mathrm{MBR}_{\mathrm{avg}}\right)$, the flow index, and the flow acceleration index $[27,28]$. LSF shows a reduction in the microcirculation in the ONH in glaucoma patients $[29,30]$. An advantage of LSF, compared with OCT-A, is the possibility to measure the waveform of the pulsatile signal, which can be changed in diseased eyes. This was shown for patients with glaucoma [28, 31].

In this paper, we describe the application of a light absorption-based method to measure the cardiac cycleinduced pulsatile changing blood volume in the $\mathrm{ONH}$ using a recently developed video ophthalmoscope. The main outcome is the photoplethysmographic signal of the tissue of the $\mathrm{ONH}$ and derived parameters, which are possibly useful as new biomarkers.

The method described here has some advantages compared with established methods. Due to the development of very light-sensitive image detectors (CCD and CMOS cameras) and compact and effective light sources (LED), such an instrument can be realized relatively simply and cost-effectively using mainly commercial components available from stock. Additionally, it can be made battery (or USB)-powered and portable $[32,33]$. The measurement (acquisition of a video sequence) is fast and non-invasive. Once the video sequence is acquired and stored, the plethysmographic signal can be calculated offline with high temporal and spatial resolutions at any position and with any size within the acquired field of view. Therefore, it is possible to assess signals from different positions along the blood vessel system consisting of arteries, arterioles, capillaries, venules, veins in the $\mathrm{ONH}$, and peripapillary area from a single measurement. An example of the comparison of signals from the entire $\mathrm{ONH}$ and a vein is given in [32]. 
Here, we concentrate on the plethysmographic signal of the capillaries of the $\mathrm{ONH}$. The proposed method was applied to 110 subjects (19 normal subjects and 91 glaucoma patients) to compare parameters of the plethysmographic signal of the $\mathrm{ONH}$ between glaucoma groups and with the retinal nerve fibre layer (RNFL) thickness assessed by optical coherent tomography (OCT).

\section{Methods}

\section{Procedures/subjects}

The study included 19 healthy subjects and 91 patients of the Erlangen Glaucoma Registry (www.clinicaltrials.gov, NCT00494923). All patients and healthy subjects had long experience with ophthalmological examinations. The Erlangen Glaucoma Registry is a clinical registry for crosssectional and longitudinal observation of patients with openangle glaucoma (OAG) or glaucoma suspect and was founded in 1991. The primary aim is the evaluation of the diagnostic and prognostic validity of morphometrical, sensory, and hemodynamic diagnostic procedures. The inclusion/exclusion criteria, the definition of the gold standard, the schedule, and the type of examinations are defined in a protocol that was approved by the local ethics committee. The study followed the tenets of the Declaration of Helsinki for research involving human subjects and informed consent, and agreements for data collection were obtained from all participants of the study. All control subjects and patients were thoroughly examined by slit-lamp inspection, applanation tonometry, fundoscopy, gonioscopy, standard automated perimetry (SAP), and papillometry. Optic disc evaluations of patients and controls were based on 15-degree colour images (Zeiss telecentric fundus camera, Carl Zeiss Meditec). Glaucomatous damage was defined as a glaucomatous appearance of the optic nerve head, e.g. an unusually small neuroretinal rim and notching, or cup/disc ratios being higher vertically than horizontally. Optic disc evaluations were independently performed by 2 glaucoma experts of the EGR. A third clinical glaucoma expert was consulted to decide in case of discrepancy [34]. In addition, a 24-h intraocular pressure curve (6 determinations) was measured in all patients. Table 1 includes the IOP at the time of video acquisition and the mean of the daily profile. The mean value of IOP did not differ between groups, with the exception of the OHT (ocular hypertension group) group, which showed slightly larger values. For some of the measurements, pupils were dilated using Mydriatikum Stulln (Pharma Stulln GmbH, Germany) and Neosynephrin (URSAPHARM Arzneimittel GmbH, Germany). Criteria for glaucoma diagnosis were an open anterior chamber angle and glaucomatous appearance of the $\mathrm{ONH}$, including an unusually small neuroretinal rim area in relation to the $\mathrm{ONH}$ size and cup-to-disc ratios being higher vertically compared with horizontal measurements [35]. All subjects of the EGR had annual examinations. During an 8-month period, all attending subjects additionally had an examination with a video ophthalmoscope.

The means of the best-corrected visual acuity (VA) in the 4 groups of the study are given in Table 1 . The minimum VAs were 0.7 in the normal and OHT groups, and 0.63 in the preperimetric glaucoma group. In the perimetric glaucoma group, there were 6 subjects (16\%) showing VAs less than 0.6 due to glaucoma. We included all subjects that were able to control fixation; refraction was compensated by the instrument.

All eyes included in the study had clear optic media. On the day of the examination, the intraocular pressure was equal to or less than $22 \mathrm{mmHg}$. Exclusion criteria were all eye diseases other than glaucoma, diabetes mellitus, colour vision anomalies, and a myopic refractive error exceeding -12 dioptres. The age was 40 years and older. The age and the pulse rate (calculated from pulse length, see below) did not differ between study groups (Kruskal-Wallis test).

\section{Perimetry}

All subjects underwent visual field testing with standard white-on-white perimetry using a computerized static projection perimeter (Octopus-G1, Interzeag, Switzerland). All patients had the 3-phase protocol (full threshold) to calculate the 'corrected loss variance' (CLV). Those subjects with rates of false-positive or false-negative responses higher than $12 \%$ were not included in this study. Similar to what has been suggested earlier [36], a white-on-white perimetry was classified as a 'non-normal' visual field when one of the following was present: (a) at least three adjacent test points in the superior or inferior hemifield having a probability of $\leq 5 \%$ and with one test point with a defect of $\leq 1 \%$, or (b) at least two adjacent test points having a probability of $\leq 1 \%$. These criteria had to be confirmed in at least the two most recent Octopus measurements at the same test location. SAP was used to separate the glaucoma cohort into perimetric and preperimetric patients.

\section{Nerve fibre layer thickness}

A spectral domain OCT (Spectralis, Heidelberg Engineering, Germany) was used to examine the mean thickness of the RNFL at a circle of 3.4-mm diameter at 768 positions around the ONH [37].

Data of the subjects in the 4 study groups are shown in Table 1.

Normal subjects The study included 19 left eyes of 19 healthy control subjects ( 9 males, 10 females). They did not show any 
abnormality in the ophthalmologic evaluation, including slitlamp inspection, perimetry, tonometry, fundoscopy, and papillometry. The controls underwent the identical diagnostic programme compared with the subjects with open-angle glaucoma and ocular hypertension.

Ocular hypertension group Patients in this group (17 left eyes; 8 males, 9 females) had earlier intraocular pressures above $21 \mathrm{mmHg}$ upon repeated measurements. All of them had a 'non-perimetric' visual field result with white-on-white perimetry and normal ONHs.

'Preperimetric' glaucoma group In the 'preperimetric' glaucoma group (24 left eyes; 11 males, 13 females), subjects showed glaucomatous abnormalities of the ONH and localized or diffuse loss of the retinal nerve fibre layer (RNFL). Computerized visual field examinations with white-on-white perimetry (Octopus, programme G1) were normal. This 'preperimetric' glaucoma group included 21 eyes with primary open-angle glaucoma and 3 eyes with secondary openangle glaucoma due to pigmentary glaucoma or pseudoexfoliation.

'Perimetric' glaucoma group The 'perimetric' glaucoma group (50 left eyes; 29 males, 21 females) included 23 eyes with primary open-angle glaucoma characterized by elevated intraocular pressure higher than $21 \mathrm{mmHg}, 15$ eyes with secondary open-angle glaucoma with elevated intraocular pressure due to pigmentary glaucoma or pseudoexfoliation, and 12 eyes with normal-pressure glaucoma. All patients of this 'perimetric' glaucoma group had glaucomatous ONH damage and local and/or diffuse visual field loss in white-on-white perimetry (mean defect $8.6 \pm 5.6$ ).

Patients with ocular hypertension and open-angle glaucoma were treated to reduce the IOP (medicamentous or with surgery, e.g. laser trabeculoplasty).

\section{Acquisition of video sequences}

The video sequences were acquired using a recently developed video ophthalmoscope $[32,33]$. The instrument is based on the principle of a fundus camera. An ophthalmic lens produces an aerial image of the retina in the aerial image plane. An objective lens images the aerial image to a light detector with high light sensitivity, either a CCD or CMOS camera. The frame rate was set to 25 frames per second. The aerial image plane contains a field stop to restrict the field of view $\left(20^{\circ} \times 15^{\circ}\right)$ centred to the $\mathrm{ONH}$ and a fixation target (small LED or OLED display) to maintain stable fixation of the subject during data acquisition. To achieve high light absorption by blood, the illuminating wavelength was $575 \mathrm{~nm}$ (LED). In the pupil plane, the illuminating and imaging light paths are 
inverted, which simplifies the optical setup [33]. The size of the entire instrument is small $(37 \mathrm{~cm} \times 7 \mathrm{~cm} \times 7 \mathrm{~cm})$.

All subjects had a 10-min rest time in the examination room before measurement to reduce the possible influence of physical exertion. After alignment of the instrument to the subject's eye (with dilated pupil) using a head and chin rest and an adapted XYZ mount of a slit lamp, 3 video sequences were acquired for each subject with a duration of $10 \mathrm{~s}$ each. The entire measurement, including alignment of the instrument, took less than $1.5 \mathrm{~min}$. The acquired video sequences were stored for off-line evaluation.

\section{Calculation of parameters}

To calculate various blood flow-related parameters (peak amplitude, steepness of ascending part of pulse, time-to-peak, full width at half maximum, and pulse duration, see below), the acquired video sequences were processed offline according to the flowchart shown in Fig. 1. The different steps are described below.

\section{Suppression of eye movements}

The video sequences must be registered to eliminate eye movements during acquisition (step 1). We have developed a method for frame-to-frame registration [38], which effectively suppresses eye shifts and rotation in acquired sequences. This method has been applied to all sequences of subjects included in this study.

The eye movements can also cause specific distortions of some frames and influence the consecutive analysis. These distortions are as follows: motion blur (due to fast eye movement during the exposure time of one frame), out-of-focus blur (due to strong optical aberration, head movement, eye focus changes), and strong eyelid reflections (due to eye blinking).

\section{Segmentation of structures}

All 250 frames of a registered sequence were averaged to create one representative retinal image (step 2), which is used for $\mathrm{ONH}$ and blood vessel segmentation. The $\mathrm{ONH}$ has been segmented manually to avoid imprecise segmentation in some challenging cases (step 3). Next, the blood vessels have been segmented automatically by a pixel-wise classification-based approach described in [39] (step 4). The segmented blood vessel tree has been further morphologically dilated (with a circular mask 5 pixels in size), which causes a slight increase of the segmented tree to eliminate the close blood vessel surroundings, which might be influenced by blood vessel pulsation and movement. These two binary masks (from $\mathrm{ONH}$ segmentation and blood vessel segmentation) are merged

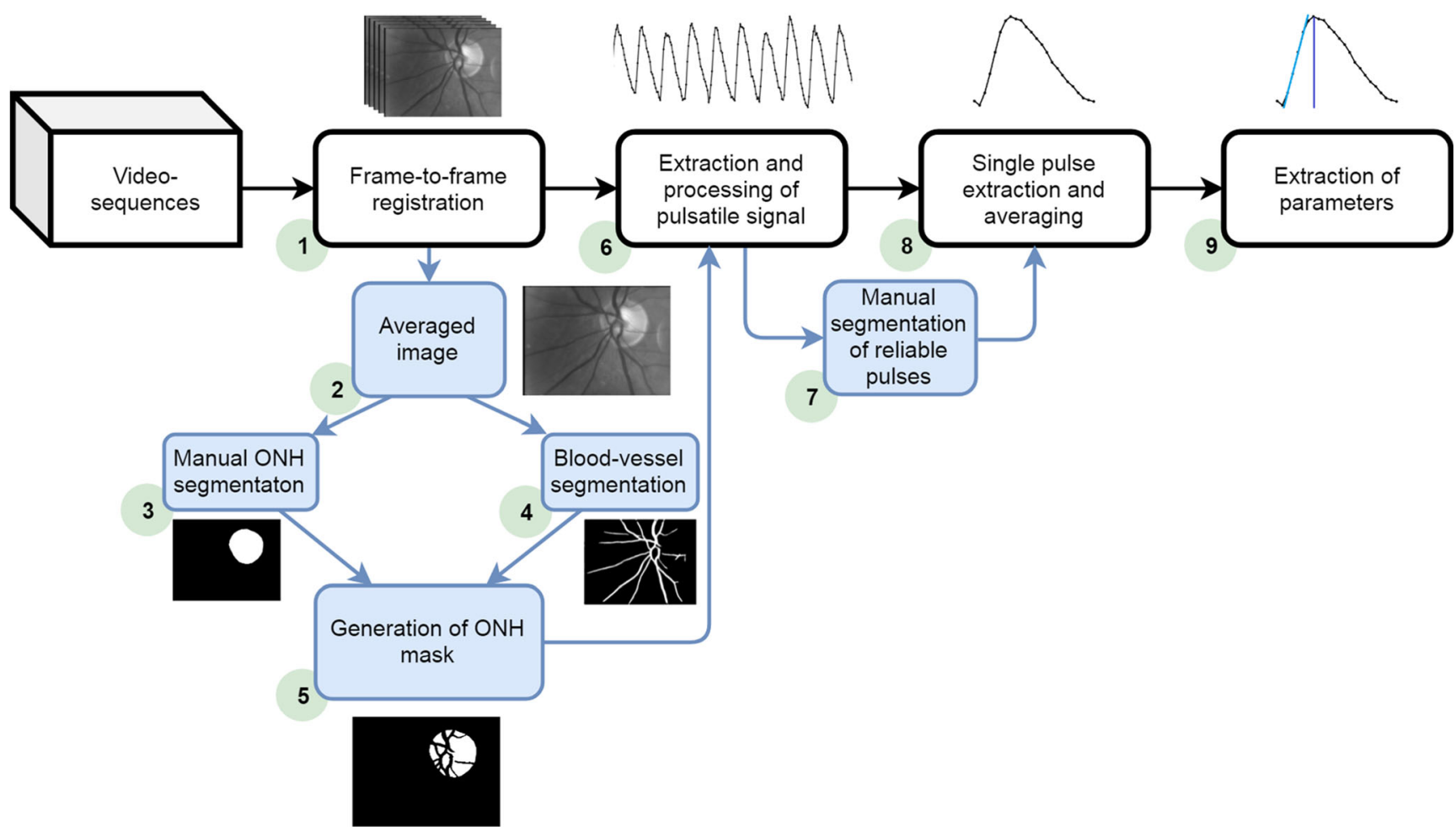

Fig. 1 Flowchart to calculate pulse parameters of the plethysmographic signal of the ONH from video sequences. The different steps (1) to (9) are explained in the text 
a
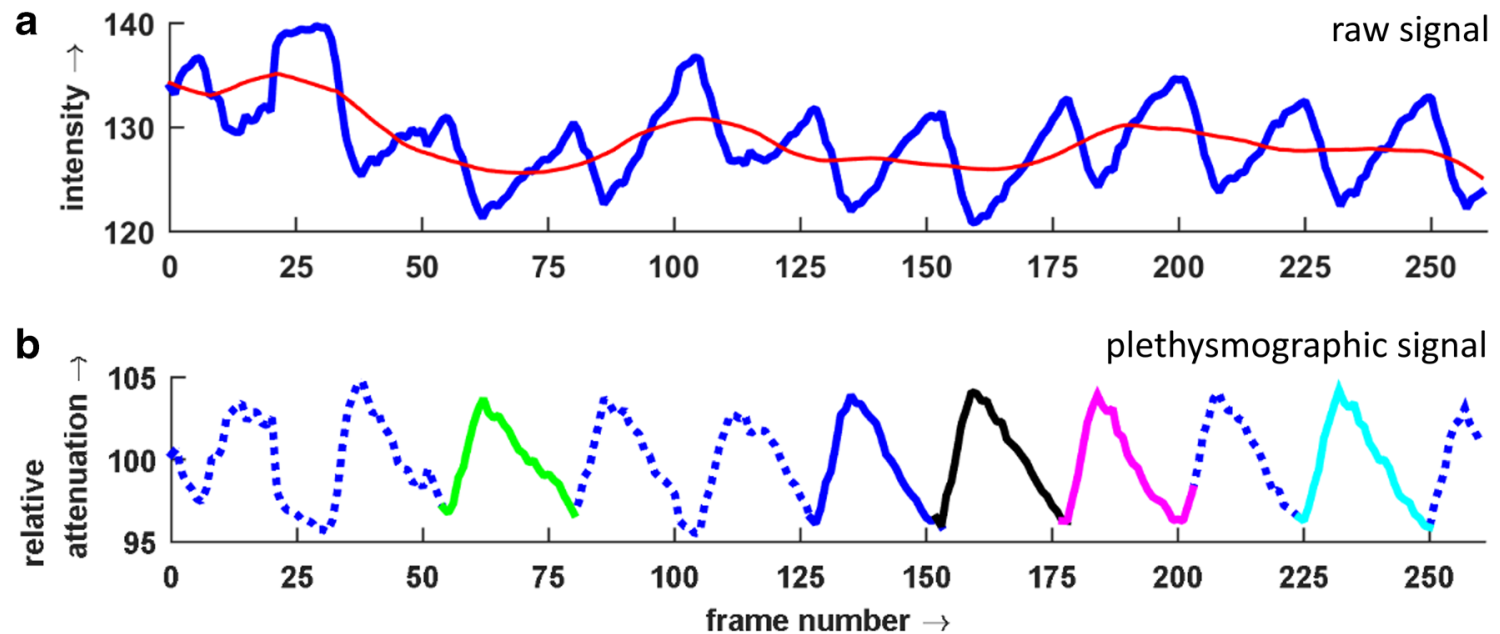

C

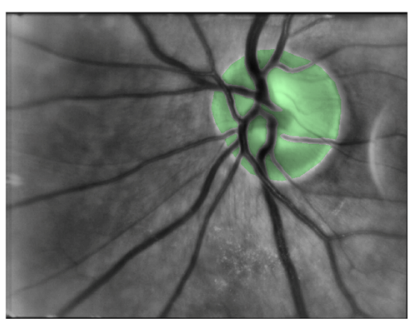

d

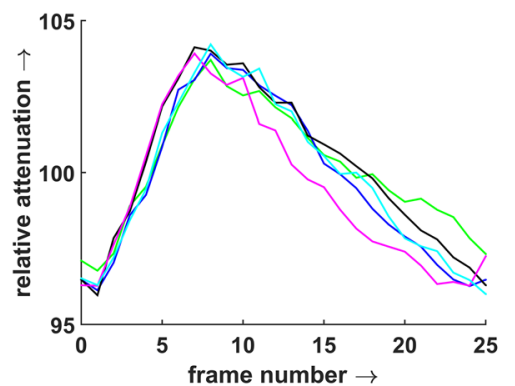

e

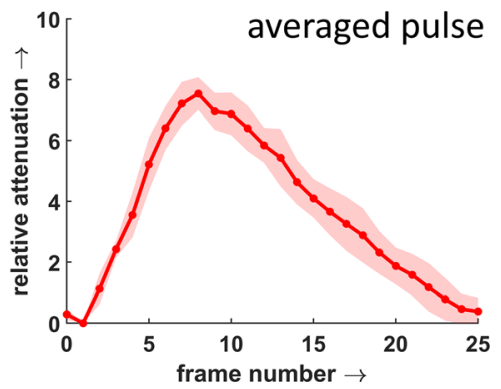

Fig. 2 Calculation of averaged pulse from raw (intensity) signal of a normal subject. (A) original pulsatile signal (thick blue line) and calculated trend (red thin line). (B) Calculated plethysmographic signal after trend correction (dotted line) and manually selected pulses (solid parts).
(C) Averaged image with segmented ONH ROI (green). (D) Five selected individual single pulses. (E) Averaged pulse was calculated from selected single pulses (red line) and standard deviation (reddish area). Twenty-five frames correspond to $1 \mathrm{~s}$ together (step 5) to create a new binary mask corresponding only to $\mathrm{ONH}$ tissue, further referred to as ONH ROI.

\section{Calculation of plethysmographic signal}

The binary mask is used to extract the reflected light intensity from ONH ROI (measured as grey levels in acquired frames) (step 6). For each frame of the sequence, the intensity for all pixels within the ONH ROI is averaged. The succession of these values of all frames of a sequence represents the signal of the changing intensity $I(n)$ ( $n$ : frame number) due to cardiac cycle-induced light absorption changes (raw signal, Fig. 2A). The raw signal is proportional to the reflected light intensity. The trend-corrected plethysmographic signal $A(n)$ that is proportional to the absorption due to the changing blood volume is calculated as $A(n)=1-\left[I(n) / I_{\text {avg }}(n)\right]$, where $I_{\text {avg }}(n)$ is the trend signal [32]. The plethysmographic signal shows higher values for higher blood volume (i.e. higher light absorption) and vice versa. To be able to directly display the results in a video sequence, all values are multiplied by 100 [32]. This signal can be interpreted as the relative deviation of light absorption from the average value (value 100) during the cardiac cycle. For instance, the value 105 means an increase of absorption of 5\% (Fig. 2B) compared with the average value.

\section{Selection of pulses}

As mentioned above, the eye movements and eye blinking during the acquisition create artefacts that cannot be completely suppressed or removed. Therefore, we decided on manual inspection of each plethysmographic signal and manual selection of reliable pulses (without distortion due to fast eye movement, eye blinks, or light reflection from corneal reflex) for parameter extraction (step 7). The investigator who selected the pulse cycles was masked to the diagnosis. The local minima of undistorted pulses have been labelled and stored for each sequence. Typically, 5 (2 to 7) pulses have been selected (Fig. 2B, D). In the next step, these labels have been used to automatically select these pulses and compute one averaged pulse (step 8). For the averaged pulse, the reference value is the starting point at the beginning of the heartbeat; this means it represents the changing light attenuation during one cardiac cycle. Further, this signal is referred to as the averaged pulse (Fig. 2E). 


\section{Pulse-related parameter extraction}

The following parameters were extracted (step 9) and tested for all averaged pulses (see Fig. 3):

- Peak amplitude in \% A - the maximum value of the averaged pulse. The unit $[\% \mathrm{~A}]$ is used to show that this signal is related to the absorption. This can be interpreted as the maximum value of blood volume with respect to the beginning of the cardiac pulse.

- Steepness of the ascending part in $\% \mathrm{~A} / \mathrm{s}$ - calculated as an increase of intensity from 30 to $70 \%$ of the value of peak amplitude divided by the corresponding time (as shown in Fig. 3). Other methods of steepness calculation (minimum-maximum value, 47 to $53 \%$ value, and maximum value of numerical differentiation) have also been tested and provided similar results. This parameter can be interpreted as the speed of blood volume increase during systole.

- Time-to-peak (s) and percentage (\%) of pulse durationtime to reach the maximum from the beginning, expressed in seconds and relatively in percentage with respect to pulse duration.

- Peak width at half maximum (FWHM) in seconds (s) and percentage $(\%)$ of pulse duration - width of the peak at $50 \%$ of its height, also computed in seconds and relatively in percentage with respect to pulse duration.

- Pulse duration in seconds (s) - duration of the averaged pulse.

\section{Statistics}

All statistical analyses were performed using SPSS software (SPSS Inc., version 21, Chicago, IL, USA). The results include means and standard deviations, as well as medians and

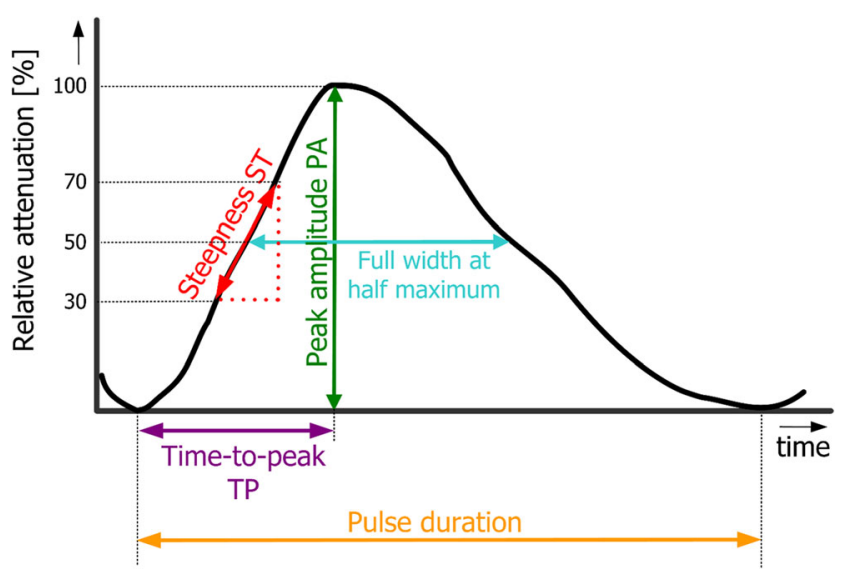

Fig. 3 Graphical explanation of calculated parameters from averaged pulse quartiles, in the boxplot presentation. The normality distribution of the results and equal variances could not be confirmed for all groups. Hence, non-parametric tests were used in the statistical analysis. Comparisons between groups were made using the Mann-Whitney $U$ test and the Kruskal-Wallis test. The Spearman correlation analysis was performed to study the association between parameters and mean thickness of RNFL. To take into account the possible error by multiple comparisons, all results were corrected according to Bonferroni. Analysis of variance was used to calculate the coefficient of variation $(\mathrm{CV})$ for two repeated measurements. $p<0.05$ was considered statistically significant for all tests.

\section{Results}

The reproducibility of the method has been evaluated using repeated measurements for 9 control subjects. The $\mathrm{CVs}$ for the pulse parameters amplitude, slope, and duration were 0.086 , 0.088 , and 0.042 , respectively.

Figure 4 shows examples of calculated averaged pulses and corresponding ONH ROI segmentation in averaged images from 3 different patient groups.

Differences in amplitude and waveform, e.g. in the behaviour of the leading and falling edges, can be clearly seen. Figure 4A shows a subject with high peak amplitude (9\%A) and a nearly linear rising edge, whereas the subjects in $\mathrm{B}$ and $\mathrm{C}$ show lower peak amplitude (about 6\%A) and deviation from the linearly rising edge.

Table 2 presents the mean values and standard deviations for all pulse parameters in the four subject groups. The table additionally includes the results ( $p$ values) comparing the groups with the Kruskal-Wallis test. Significant results $(p<$ 0.001 ) were found for steepness and peak amplitude. However, time-to-peak, FWHM, and pulse duration did not differ between groups.

For the parameters with significant differences between groups (peak amplitude and steepness, see Table 2), boxplots including medians and quartiles are presented in Fig. 5. The figures clearly show reductions of peak amplitude and steepness in the more advanced glaucoma groups.

The relationship between the pulse parameters and the severity of glaucoma disease is furthermore shown by correlation analyses for the total patient cohort. The correlation analyses (Spearman test) of mean thickness of RNFL with the pulse parameters revealed significant correlation coefficients $(p<0.001)$ for peak amplitude $(R=0.523)$ and steepness $(R=0.568)$, but not for time-to-peak, FWHM, and pulse duration (not shown graphically). Separate correlation analyses in males and females showed similar relationships between slope and RNFL thickness for both groups (male $R=0.31$, female $R=0.29$ ).

The scatterplots showing peak amplitude and steepness as functions of RNFL mean thickness are presented in Fig. 6. 

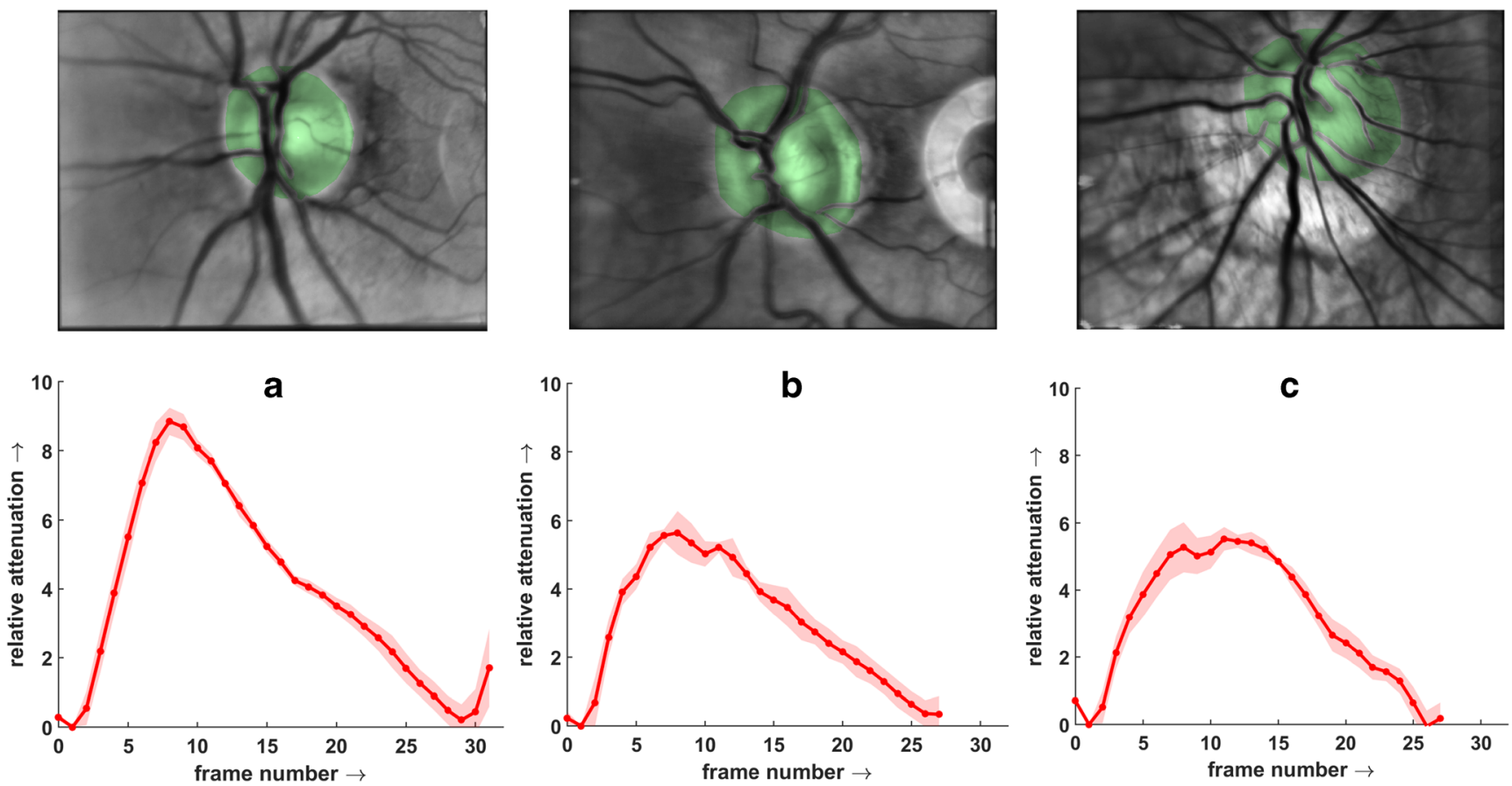

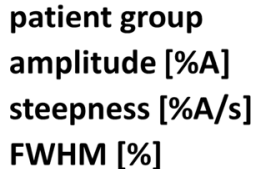

$\mathrm{OHT}$

9.4

41

43

Fig. 4 Examples of calculated averaged pulses. Upper row: averaged fundus images of the entire sequence and segmented ONH ROIs (green area). Middle row: averaged pulses (red line) and standard deviations (reddish areas) calculated from the green ROIs shown in the upper row. Lower row: calculated pulse parameters (the bright semicircle on the right

Regression lines indicate reductions of peak amplitude by $0.6 \% \mathrm{~A}$ and steepness by $3 \% \mathrm{~A} / \mathrm{sec}$ for every $10-\mu \mathrm{m}$ loss of RNFL thickness.

\section{Discussion}

The method described in this paper measures the pulsatile component of the changing blood volume in the capillaries of the ONH tissue. Our results show reductions of peak

prep
5.7
28
50

perim

5.6

22

56

side in image $\mathrm{B}$ is due to a not correct aligned aperture during this measurement, but it has no influence on the result.) A corresponding example of a normal subject (amplitude $7.6 \% \mathrm{~A}$, steepness $34 \% \mathrm{~A} / \mathrm{s}$, FWHM 44\%) is shown in Fig. 2C and E. Patient groups: OHT, ocular hypertension; prep, preperimetric glaucoma; perim, perimetric glaucoma

amplitude and steepness with increasing severity of glaucoma and a high correlation of these two parameters with RNFL thickness. The peak amplitude can be related to the amount of pulsatile blood volume flowing through examined tissue and depends on both tissue capillary density and the amount of blood flowing through these capillaries. The steepness of our pulsatile component can be interpreted as the velocity of blood filling, i.e. how fast the tissue is filled by blood during systole. Our findings show a reduction of the values of these two parameters in glaucoma subjects. Although our pulse

Table 2 Mean, standard deviation, and outcome of the Kruskal-Wallis test for parameters in different subject groups. Patient groups: OHT, ocular hypertension; prep, preperimetric glaucoma; perim, perimetric glaucoma; norm, normal subjects

\begin{tabular}{lllllllll}
\hline Patient group & $\begin{array}{l}\text { Number of Peak amplitude } \\
\text { subjects }\end{array}$ & $\begin{array}{l}\text { Steepness } \\
(\% \mathrm{~A} / \mathrm{sec})\end{array}$ & Time to peak (s) & Time to peak (\%) & FWHM (s) & FWHM (\%) & Pulse duration (s) \\
\hline Norm & 19 & $5.86 \pm 1.84$ & $29.23 \pm 6.72$ & $0.34 \pm 0.09$ & $33.09 \pm 7.67$ & $0.56 \pm 0.12$ & $50.29 \pm 8.93$ & $1.05 \pm 0.18$ \\
OHT & 17 & $6.60 \pm 2.41$ & $32.55 \pm 9.98$ & $0.30 \pm 0.05$ & $32.25 \pm 7.30$ & $0.51 \pm 0.08$ & $50.68 \pm 7.16$ & $0.94 \pm 0.16$ \\
Prep & 24 & $5.17 \pm 1.80$ & $24.27 \pm 9.33$ & $0.35 \pm 0.08$ & $32.54 \pm 5.29$ & $0.55 \pm 0.12$ & $47.28 \pm 7.72$ & $1.08 \pm 0.13$ \\
Perim & 50 & $4.06 \pm 1.67$ & $18.84 \pm 8.26$ & $0.35 \pm 0.08$ & $35.88 \pm 7.27$ & $0.55 \pm 0.09$ & $51.86 \pm 7.61$ & $1.00 \pm 0.17$ \\
Kruskal-Wallis test & $p$ & $<0.001$ & $<0.001$ & n.s. & n.s. & n.s. & n.s. & n.s. \\
\hline
\end{tabular}




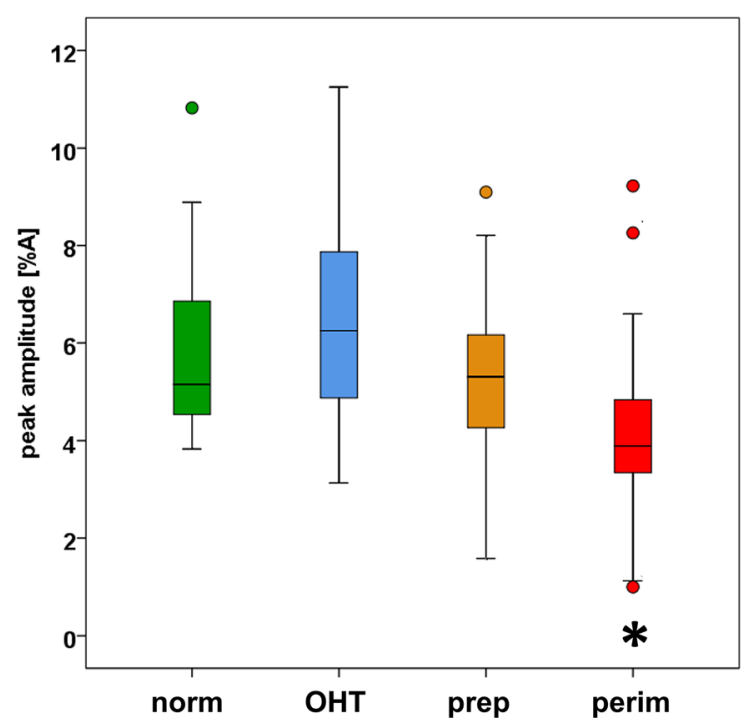

Fig. 5 Boxplots showing medians and quartiles of peak amplitude and steepness for the different subject groups. Significant differences $(p<$ 0.001 ) between groups (Mann-Whitney test) in comparison with normal

parameters are based on light absorption related to blood volume changes and not on particle movement, we showed that our first results of $\mathrm{ONH}$ perfusion assessment are in agreement with the results obtained by other, more sophisticated modalities such as OCT-A and LSF:

Using OCT-A, Jia et al. 2012 [16] showed large flow index and vessel density reduction for three preperimetric glaucoma subjects, particularly in temporal regions of the ONH. Significant ONH perfusion reduction has also been found by Chen et al. [21] in glaucomatous eyes using optical microangiography (OMAG)-derived ONH perfusion metrics (blood flux, vessel density area, normalized flux) from

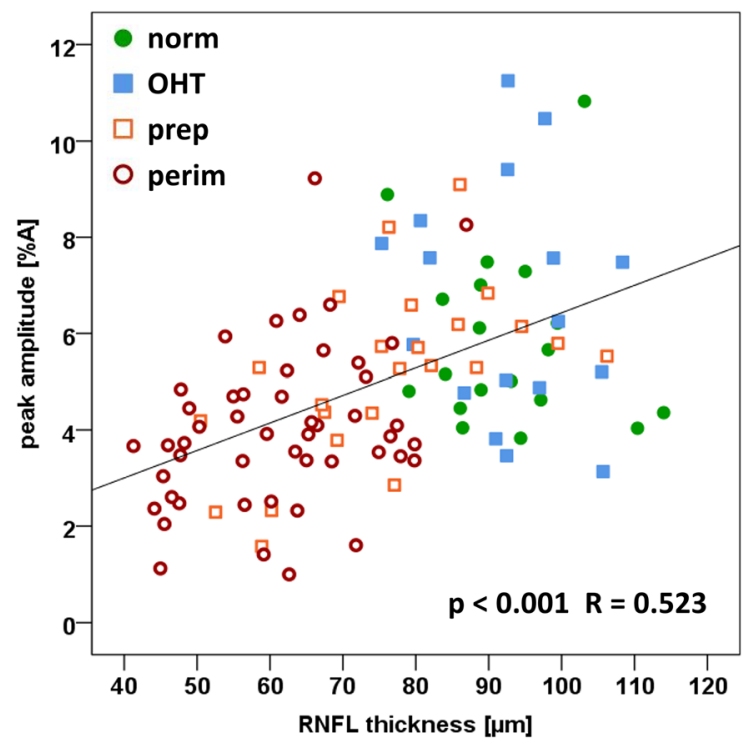

Fig. 6 Scatterplots of peak amplitude and steepness as functions of RNFL mean thickness, including regression lines. The Spearman test indicates a significant association between peak amplitude and

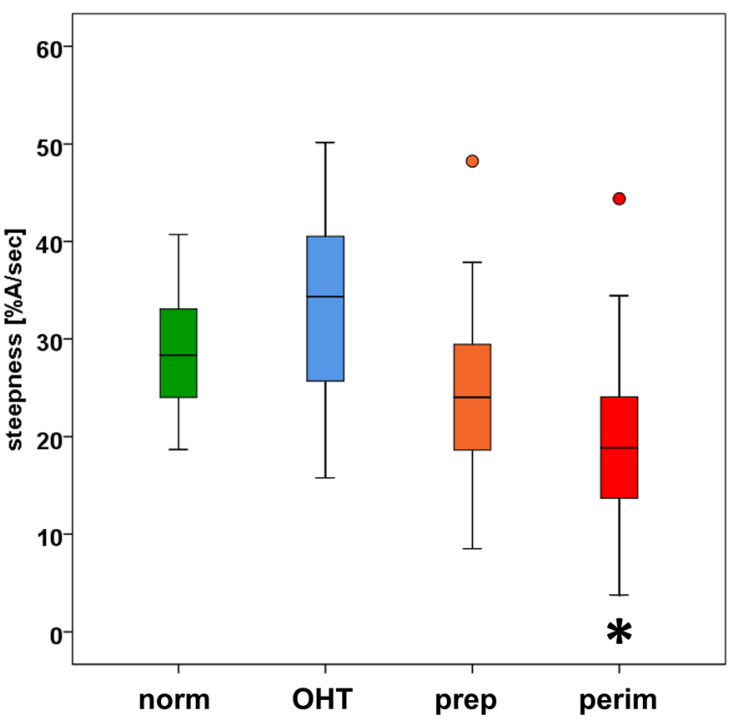

subjects are indicated by asterisks $(*)$. Patient groups: OHT, ocular hypertension; prep, preperimetric glaucoma; perim, perimetric glaucoma; norm, normal subjects

prelaminar tissues (tissues between the internal limiting membrane and the anterior surface of the laminar cribrosa). Significant negative correlations between ONH perfusion and glaucoma severity have also been found to be statistically significant. Wang et al. [22] explored ONH perfusion in openangle glaucoma (OAG) subjects and found a statistically significant correlation between RNFL thickness and ONH flow index in OAG subjects $(n=62)$, but not in healthy subjects $(n$ $=20$ ). The mean RNFL thicknesses in mild, moderate, and severe glaucoma groups were $86.5 \mu \mathrm{m}, 77.0 \mu \mathrm{m}$, and 64.0 $\mu \mathrm{m}$, respectively, corresponding to the RNFL thickness in our study. Lévêque et al. [40] also found this statistically

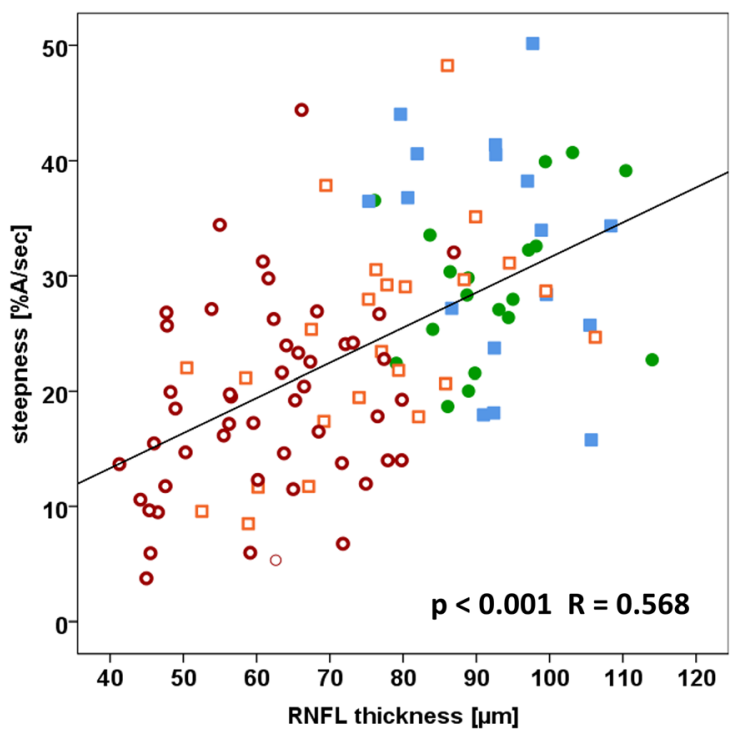

steepness with RNFL thickness. Patient groups: OHT, ocular hypertension; prep, preperimetric glaucoma; perim, perimetric glaucoma; norm, normal subjects 
significant correlation (RNFL thickness vs. blood vessel density in both, whole and temporal ONH part) for advanced glaucoma cases included in their study (mean RNFL value $66.48 \pm 12.6 \mu \mathrm{m}$ ). A comprehensive review of OCT-A in glaucoma is given in [41], where authors reviewed various studies suggesting that OCT-A-derived perfusion parameters decrease in $\mathrm{ONH}$, peripapillary area, and macular area.

Laser speckle flowgraphy is another modality used to assess ONH perfusion. Although a recent comparison [42] of OCT-A and LSF showed that vessel density (from OCT-A) has a higher glaucoma diagnostic ability compared with LSF parameters in patients with normal tension glaucoma (NTG), the advantage of LSF is that it has an additionally high temporal resolution. This enables analysis of speckle-induced pulsatile changes, i.e. shape of the waveforms from different retinal regions, on a pulse-by-pulse level. Recent LSF-based studies describe changes of these waveforms (and related parameters) for glaucoma subjects. Shiga et al. [31] described a delay in the peak of the MBR waveform, a flattened waveform, and reduced MBR amplitude for normal tension glaucoma (NTG) subjects in the ONH capillary area only. MurschEdlmayr et al. [28] found a reduced blood flow in the vessels supplying the retina assessed by MBR from ONH in NTG subjects. They also evaluated the shape of the MBR curve using several specific parameters and found a flatter waveform for NTG subjects, which means a lower $\mathrm{MBR}_{\mathrm{avg}}$ and a lower flow acceleration index. The results of these studies are similar to our findings - lower waveform amplitude and lower steepness in glaucoma groups. Although the results from different studies are not coherent, it is evident that the waveform analysis of blood-induced pulsatile patterns extracted from $\mathrm{ONH}$ capillaries and obtained by different principles can contribute to an improved understanding of glaucoma vascular dysfunction. Furthermore, there are studies (e.g. [29]) showing that MBR from the $\mathrm{ONH}$ capillaries is significantly correlated with the RNFL thickness in the superior, inferior, and temporal ONH quadrants.

The waveform of the pulsatile signal contains important information. It can be affected by different kinds of provocation, such as increased intraocular pressure [43] and flicker light stimulation [44, 45], and also by diseases, such as carotid artery stenosis (CAS) [46-50] and glaucoma [31, 51]. Some of the above-mentioned results are based on the measurement of the pulsatile signal of the intraocular pressure and not on the blood volume absorption signal. However, it was shown for the capillaries of the finger that the blood volume pulse and the radial pressure pulse can be represented by a single mathematical transfer function. This implies that the information in the blood volume pulse is similar to that contained in the radial pressure pulse [52], so that it has to be expected that changes in the IOP pulse can also be seen in the blood volume pulse.

OBF can be affected by IOP, blood pressure, heart rate, systemic diseases, cardiovascular diseases, medication (especially antihypertensive and hypotensive medication, topical medication), haemoglobin concentration, haematocrit, and vessel stiffness. However, the fact that most of the above-mentioned parameters are naturally identical on both eye sides allows the detection of differences that are caused by asymmetric IOP and/or asymmetric stenosis of carotid arteries (CAS). As diseases such as glaucoma [53-55] and CAS often start asymmetrically [50], the exact comparison of the parameters of the plethysmographic signals between eye sides could reveal deviation from normal behaviour caused by beginning CAS and/or increased IOP as a sign of beginning glaucoma. Using a binocular video ophthalmoscope (consisting of two identical video ophthalmoscopes as described here), it is possible to measure both eyes simultaneously [32]. Providing an exact synchronization of the cameras of the two instruments, parameters of the plethysmographic signal that are caused by the same heartbeat can be exactly compared. In this way, even a small time shift between sides can be measured. The first results show that, for instance, the plaque of the carotis communis can cause a small time delay between eye sides [32]. Therefore, it could be possible to detect pathologic differences between the signals on both eye sides without knowledge of the other OBF-influencing parameters.

Nevertheless, a possible limitation of our study is that many parameters that can influence OBF are not considered. However, at the present state of our development, there are too few subjects to draw reliable clinical results on the influence of these parameters.

Future work will involve measuring the influence of the above-mentioned OBF-related parameters on the plethysmography signal in a larger subject group and also the comparison of the plethysmographic signals of right and left eyes to detect deviation from symmetry as an early sign of the initiation of diseases such as glaucoma or carotid artery stenosis.

\section{Conclusion}

The presented low-cost video-ophthalmoscope allows the measurement of the plethysmographic signal of the ONH tissue and the calculation of different perfusion-related parameters. Our findings show that the values of peak amplitude and steepness are significantly reduced in patients with perimetric glaucoma in comparison with normal subjects, and both parameters showed significant correlations with RNFL thickness. This is in line with the results of other clinical techniques such as OCT angiography and laser speckle flowmetry, which also showed reduced blood flow in ONH tissue in glaucoma. A possible limitation of our study is that the influences of OBF-related parameters such as blood pressure and heart rate on these measurements are not considered. The influence of the latter parameters might be lower if simultaneous 
measurements of the right and left eyes would be compared to detect deviations from symmetry. Due to the small size of the instrument, it is possible to use two video-ophthalmoscopes to measure both eyes simultaneously. This binocular setting will allow comparisons of the plethysmographic signals of both eye sides induced by the same heartbeat and quantification of the asymmetry of ONH blood flow among both eyes with high spatial and temporal resolution.

Acknowledgements We thank the staff of the Erlangen Glaucoma Registry for their valuable clinical support and substantial information.

Funding Open Access funding enabled and organized by Projekt DEAL. This study was supported by the Deutsche Forschungsgemeinschaft DFG (TO 115/3-1) to RPT.

Data availability Not applicable

\section{Compliance with ethical standards}

Conflicts of interest The authors declare that they have no conflicts of interest.

Ethics approval The study was approved by the ethics committee of the Friedrich-Alexander-Universität Erlangen-Nürnberg (No. 4400) and complies with the tenets of the Declaration of Helsinki.

Consent to participate Informed consent was obtained from all subjects in the study, including the consent to publish the data in a journal.

\section{Consent for publication See above}

Code availability Not applicable

Open Access This article is licensed under a Creative Commons Attribution 4.0 International License, which permits use, sharing, adaptation, distribution and reproduction in any medium or format, as long as you give appropriate credit to the original author(s) and the source, provide a link to the Creative Commons licence, and indicate if changes were made. The images or other third party material in this article are included in the article's Creative Commons licence, unless indicated otherwise in a credit line to the material. If material is not included in the article's Creative Commons licence and your intended use is not permitted by statutory regulation or exceeds the permitted use, you will need to obtain permission directly from the copyright holder. To view a copy of this licence, visit http://creativecommons.org/licenses/by/4.0/.

\section{References}

1. Flammer J, Orgül S, Costa VP et al (2002) The impact of ocular blood flow in glaucoma. Prog Retin Eye Res 21:359-393

2. Schubert G (1936) Untersuchung des Blutsauerstoffgehaltes und der Durchblutung des Auges auf lichtelektrischem Wege. Albrecht Von Graefes Arch Ophthalmol 135:558-560. https://doi. org/10.1007/BF01853424

3. Trokel S (1964) Measurement of ocular blood flow and volume by reflective densitometry. Arch Ophthalmol 71:88-92
4. Trokel S (1964) Photometric study of ocular blood flow in man. Arch Ophthalmol 71:528-530

5. Beintema DK, Mook GA, Worst JGF (1964) Recording of arm-toretina circulation-time by means of fundus reflectometry. Ophthalmologica 148:163-168

6. Matsuo H, Kogure F, Takahasi K (1966) Studies of the photoelectric plethysmogram of the eye. Procceedings XX Int Congr Ophthalmol 1966 178-182

7. Tornow RP, Kopp O, Schultheiss B (2003) Time course of fundus reflection changes according to the cardiac cycle. In: Invest. Ophthalmol. Vis. Sci. pp 1296-ARVO Abstract

8. Tornow RP, Kopp O (2006) Time course and frequency spectrum (0 to $12,5 \mathrm{~Hz}$ ) of fundus reflection. In: Invest. Ophthalmol. Vis. Sci. pp 3753-ARVO Abstract

9. Lovasik JV, Gagnon M, Kergoat H (1994) A novel noninvasive videographic method for quantifying changes in the chromaticity of the optic nerve head with changes in the intraocular pressure, pulsatile choroidal blood flow and visual neural function in humans. Surv Ophthalmol 38(Suppl):S35-S51

10. Morgan WH, Hazelton ML, Betz-Stablein BD et al (2014) Photoplethysmographic measurement of various retinal vascular pulsation parameters and measurement of the venous phase delay. Investig Ophthalmol Vis Sci 55:5998-6006. https://doi.org/10. 1167/iovs.14-15104

11. Hassan H, Jaidka S, Dwyer VM, Hu S (2018) Assessing blood vessel perfusion and vital signs through retinal imaging photoplethysmography. Biomed Opt Express 9:2351. https://doi. org/10.1364/boe.9.002351

12. Briers JD (1996) Laser Doppler and time-varying speckle: a reconciliation. J Opt Soc Am A 13:345. https://doi.org/10.1364/josaa.13. 000345

13. Briers JD (2001) Laser Doppler, speckle and related techniques for blood perfusion mapping and imaging. Physiol Meas 22:R35-R66. https://doi.org/10.1088/0967-3334/22/4/201

14. Michelson G, Schmauss B (1995) Two dimensional mapping of the perfusion of the retina and optic nerve head. Br J Ophthalmol 79: 1126-1132. https://doi.org/10.1136/bjo.79.12.1126

15. Wang RK, Jacques SL, Ma Z et al (2007) Three dimensional optical angiography. Opt Express 15:4083. https://doi.org/10.1364/oe.15. 004083

16. Jia Y, Morrison JC, Tokayer J et al (2012) Quantitative OCT angiography of optic nerve head blood flow. Biomed Opt Express 3: 3127. https://doi.org/10.1364/boe.3.003127

17. Zhang A, Zhang Q, Chen C-L, Wang RK (2015) Methods and algorithms for optical coherence tomography-based angiography: a review and comparison. J Biomed Opt 20:100901. https://doi.org/ 10.1117/1.jbo.20.10.100901

18. Gao SS, Jia Y, Zhang M et al (2016) Optical coherence tomography angiography. Investig Ophthalmol Vis Sci 57:OCT27-OCT36. https://doi.org/10.1167/iovs.15-19043

19. Hagag AM, Gao SS, Jia Y, Huang D (2017) Optical coherence tomography angiography: technical principles and clinical applications in ophthalmology. Taiwan J Ophthalmol 7:115-129. https:// doi.org/10.4103/tjo.tjo_31_17

20. Gräfe MGO, Gondre $\bar{M}$, de Boer JF (2019) Precision analysis and optimization in phase decorrelation OCT velocimetry. Biomed Opt Express 10:1297. https://doi.org/10.1364/boe.10.001297

21. Chen C-L, Bojikian KD, Gupta D et al (2016) Optic nerve head perfusion in normal eyes and eyes with glaucoma using optical coherence tomography-based microangiography. Quant Imaging Med Surg 6:125. https://doi.org/10.21037/QIMS.2016.03.05

22. Wang X, Jiang C, Ko T et al (2015) Correlation between optic disc perfusion and glaucomatous severity in patients with open-angle glaucoma: an optical coherence tomography angiography study. Graefes Arch Clin Exp Ophthalmol 253:1557-1564. https://doi. org/10.1007/s00417-015-3095-y 
23. Rao HL, Kadambi SV, Weinreb RN et al (2017) Diagnostic ability of peripapillary vessel density measurements of optical coherence tomography angiography in primary open-angle and angle-closure glaucoma. Br J Ophthalmol 101. https://doi.org/10.1136/ bjophthalmol-2016-309377

24. Chihara E, Dimitrova G, Amano H, Chihara T (2017) Discriminatory power of superficial vessel density and prelaminar vascular flow index in eyes with glaucoma and ocular hypertension and normal eyes. Investig Ophthalmol Vis Sci 58:690-697. https:// doi.org/10.1167/iovs.16-20709

25. Lommatzsch C, Rothaus K, Koch JM et al (2018) Vessel density in OCT angiography permits differentiation between normal and glaucomatous optic nerve heads. Int J Ophthalmol 11:835-843. https://doi.org/10.18240/ijo.2018.05.20

26. Konishi N, Tokimoto Y, Kohra K, Fujii H (2002) New laser speckle flowgraphy system using CCD camera. Opt Rev. https://doi.org/10. 1007/s10043-002-0163-4

27. Luft N, Wozniak PA, Aschinger GC et al (2016) Ocular blood flow measurements in healthy white subjects using laser speckle flowgraphy. PLoS One 11:e0168190. https://doi.org/10.1371/ journal.pone. 0168190

28. Mursch-Edlmayr AS, Luft N, Podkowinski D et al (2018) Laser speckle flowgraphy derived characteristics of optic nerve head perfusion in normal tension glaucoma and healthy individuals: a Pilot study. Sci Rep 8:5343. https://doi.org/10.1038/s41598-018-231490

29. Yokoyama Y, Aizawa N, Chiba N et al (2011) Significant correlations between optic nerve head microcirculation and visual field defects and nerve fiber layer loss in glaucoma patients with myopic glaucomatous disk. Clin Ophthalmol 5:1721-1727. https://doi.org/ 10.2147/OPTH.S23204

30. Shiga Y, Kunikata H, Aizawa N et al (2016) Optic nerve head blood flow, as measured by laser speckle flowgraphy, is significantly reduced in preperimetric glaucoma. Curr Eye Res 41:1447-1453. https://doi.org/10.3109/02713683.2015.1127974

31. Shiga Y, Omodaka K, Kunikata H et al (2013) Waveform analysis of ocular blood flow and the early detection of normal tension glaucoma. Investig Ophthalmol Vis Sci 54:7699-7706. https:// doi.org/10.1167/iovs.13-12930

32. Tornow R-P, Odstrcilik J, Kolar R (2018) Time-resolved quantitative inter-eye comparison of cardiac cycle-induced blood volume changes in the human retina. Biomed Opt Express 9:6237. https:// doi.org/10.1364/boe.9.006237

33. Tornow RP, Kolar R, Odstrcilik J (2015) Non-mydriatic video ophthalmoscope to measure fast temporal changes of the human retina. In: Progress in Biomedical Optics and Imaging - Proceedings of SPIE. p 954006

34. Jonas JB, Gusek GC, Naumann GOH (1988) Optic disc morphometry in chronic primary open-angle glaucoma - I. Morphometric intrapapillary characteristics. Graefes Arch Clin Exp Ophthalmol. https://doi.org/10.1007/BF02169199

35. Jonas JB, Budde WM, Panda-Jonas S (1999) Ophthalmoscopic evaluation of the optic nerve head. Surv Ophthalmol. https://doi. org/10.1016/S0039-6257(98)00049-6

36. Skuta GL (1992) Automated static perimetry. Am J Ophthalmol 114:110-111. https://doi.org/10.1016/s0002-9394(14)77431-8

37. Bendschneider D, Tornow RP, Horn FK et al (2010) Retinal nerve fiber layer thickness in normals measured by spectral domain oct. J Glaucoma 19. https://doi.org/10.1097/JJG.0b013e3181c4b0c7

38. Kolar R, Tornow RP, Odstrcilik J, Liberdova I (2016) Registration of retinal sequences from new video-ophthalmoscopic camera. Biomed Eng Online 15:57. https://doi.org/10.1186/s12938-0160191-0

39. Odstrcilik J, Kolar R, Harabis V, Tornow RP (2015) Classificationbased blood vessel segmentation in retinal images. In:
Computational Vision and Medical Image Processing V. CRC Press, pp 95-100

40. Lévêque P-MM, Zéboulon P, Brasnu E et al (2016) Optic disc vascularization in glaucoma: value of spectral-domain optical coherence tomography angiography. J Ophthalmol 2016:1-9. https:// doi.org/10.1155/2016/6956717

41. Van Melkebeke L, Barbosa-Breda J, Huygens M, Stalmans I (2018) Optical coherence tomography angiography in glaucoma: a review. Ophthalmic Res. https://doi.org/10.1159/000488495

42. Takeyama A, Ishida K, Anraku A et al (2018) Comparison of optical coherence tomography angiography and laser speckle flowgraphy for the diagnosis of normal-tension glaucoma. J Ophthalmol 2018:1-9. https://doi.org/10.1155/2018/1751857

43. Lawrence C, Schlegel WA (1966) Ophthalmic pulse studies. I. Influence of intraocular pressure. Investig Ophthalmol 5:515-525

44. Michelson G, Patzelt A, Harazny J (2002) Flickering light increases retinal blood flow. In: Retina, 2002/06/11. pp 336-343

45. Crittin M, Riva CE (2004) Functional imaging of the human papilla and peripapillary region based on flicker-induced reflectance changes. Neurosci Lett 360:141-144. https://doi.org/10.1016/j. neulet.2004.02.063

46. Best M, Plechaty G, Harris L, Galin MA (1971) Ophthalmodynamometry and ocular pulse studies in carotid occlusion. Arch Ophthalmol 85:334-338

47. Perkins ES (1985) The ocular pulse and intraocular pressure as a screening test for carotid artery stenosis. Br J Ophthalmol 69:676 680

48. Kinsner W, Yan Y (1990) A model of the carotid vascular system with stenosis at the carotid bifurcation. Math Comput Model 14: 582-585. https://doi.org/10.1016/0895-7177(90)90249-M

49. Rina M, Shiba T, Takahashi M et al (2015) Pulse waveform analysis of optic nerve head circulation for predicting carotid atherosclerotic changes. Graefes Arch Clin Exp Ophthalmol. https://doi. org/10.1007/s00417-015-3123-y

50. Knecht PB, Menghini M, Bachmann LM et al (2012) The ocular pulse amplitude as a noninvasive parameter for carotid artery stenosis screening: a test accuracy study. Ophthalmology 119:1244 1249. https://doi.org/10.1016/j.ophtha.2011.12.040

51. Pinto LA, Vandewalle E, de Clerck E et al (2012) Ophthalmic artery Doppler waveform changes associated with increased damage in glaucoma patients. Investig Ophthalmol Vis Sci 53:24482453. https://doi.org/10.1167/iovs.11-9388

52. Millasseau SC, Guigui FG, Kelly RP et al (2000) Noninvasive assessment of the digital volume pulse. Comparison with the peripheral pressure pulse. Hypertens (Dallas, Tex 1979) 36:952-956. https://doi.org/10.1161/01.hyp.36.6.952

53. Levine RA, Demirel S, Fan J et al (2006) Asymmetries and visual field summaries as predictors of glaucoma in the ocular hypertension treatment study. Invest Ophthalmol Vis Sci 47:3896-3903. https://doi.org/10.1167/iovs.05-0469

54. Sullivan-Mee M, Ruegg CC, Pensyl D et al (2013) Diagnostic precision of retinal nerve fiber layer and macular thickness asymmetry parameters for identifying early primary open-angle glaucoma. Am J Ophthalmol 156:567-577.e1. https://doi.org/10.1016/j. ajo.2013.04.037

55. Hou H, Moghimi S, Zangwill LM et al (2018) Inter-eye asymmetry of optical coherence tomography angiography vessel density in bilateral glaucoma, glaucoma suspect, and healthy eyes. Am J Ophthalmol 190:69-77. https://doi.org/10.1016/j.ajo.2018.03.026

Publisher's note Springer Nature remains neutral with regard to jurisdictional claims in published maps and institutional affiliations. 